\title{
Pre-sharpened and Flat-top Microtip Coupons: a Quantitative Comparison for Atom-Probe Analysis Studies
}

\author{
Keith Thompson, David J. Larson, Robert Ulfig \\ Imago Scientific Instruments, Inc. 6300 Enterprise Ln. Suite 100; Madison, WI 53711
}

The implementation of a local electrode in 3-D Atom Probe analysis [1], allows the use of flat-top and pre-sharpened microtip coupons as a sample preparation vehicle. Microtip coupons offer numerous advantages over traditional "dense break-out and mount" techniques [2]. The presence of several dozen or more microtips per coupon provides the potential for a statistically significant number of data sets in a short amount of time, under identical processing conditions, without the need to cycle new specimens into the vacuum chamber. The coupon shown in Fig. 1, for example, contains 81 microtips, all oriented normal to the wafer surface. This provides a common eucentric and offers two additional advantages: (1) atom-probe progression through multilayer films is normal to the wafer surface, Fig. 2; and (2) it minimizes ion beam alignment between individual specimens during any required focused ion beam (FIB) sharpening process. "Pre-sharpened" microtips, with a tip radius of curvature $\sim 50 \mathrm{~nm}$, offer an additional advantage in that they do not require FIB sharpening. Elimination of the costly and time consuming FIB process alleviates the detriments associated with Ga contamination and "knock-on", or ion-mixing, damage of the stack during FIB sharpening [3]. Furthermore, the samples are ready for analysis immediately upon completion of stack deposition. With collection rates of up to 20 million ions/hour, 3D-LEAP analysis of stacks deposited on microtips yield statistically relevant stack quantification within hours of deposition [4].

The difference in flat-top and pre-sharpened microtips in atom-probe analysis was investigated by analyzing the following stack: $\mathrm{Si}(\mathrm{sub}) / \mathrm{M} 1 / \mathrm{M} 1-\mathrm{M} 2 / \mathrm{M} 2-(\mathrm{sub}) / 10 \mathrm{~nm} / 60 \mathrm{~nm} / 5 \mathrm{~nm}$ (where $\mathrm{M}$ denotes "metal" film). The flat-top posts were capped with $30 \mathrm{~nm}$ of Ni and FIB sharpened to a 100 $\mathrm{nm}$ tip diameter. The pre-sharpened microtips were loaded directly into the atom probe. A 3-D image of the stack obtained from the pre-sharpened microtips is shown in Fig. 3. A magnified image of the Ni FIB cap is shown in Fig. 4, which shows that the FIB cap adequately protected the stack from Ga penetration. Concentration profiles of the two stacks are shown in Fig. 5. Quantitative analysis shows that the data collected is statistically the same, with the exception of the Ga damage present in the Ni cap of the flat-top sample. Both coupon types (1) resolved the presence of a thin M1-oxide layer at the (M1-M2)-M1 interface; (2) quantified the random distribution of C atoms (not shown) throughout the stack; (3) detailed the atomic composition of the (M1-M2) layer; and (4) accurately measured the thickness of each layer. Given the statistical similarity in data analysis, the pre-sharpened microtip coupons provide a superior analysis vehicle since Ga damage from the FIB sharpening process is eliminated and turn-around time to data (and learning) is reduced to a few hours after stack deposition. If one is concerned with subtle deviations that may be introduced by the radius of curvature of pre-sharpened tips, then FIB sharpening and analysis of analogous flat-topped microtips can verify the validity of the data rapidly collected from the pre-sharpened microtips.

[1] T.F. Kelly and D.J. Larson, Mat. Char. 44(1/2) (Feb. 2000) 59.

[2] D. J. Larson et al., Microsc. and Microanal. 7 (2001) 24.

[3] D. J. Larson et. al., Ultramicroscopy 79 (1999) 287.

[4] T.F. Kelly et. al. Microsc. and Microanal. 10(3) (2004) 373. 


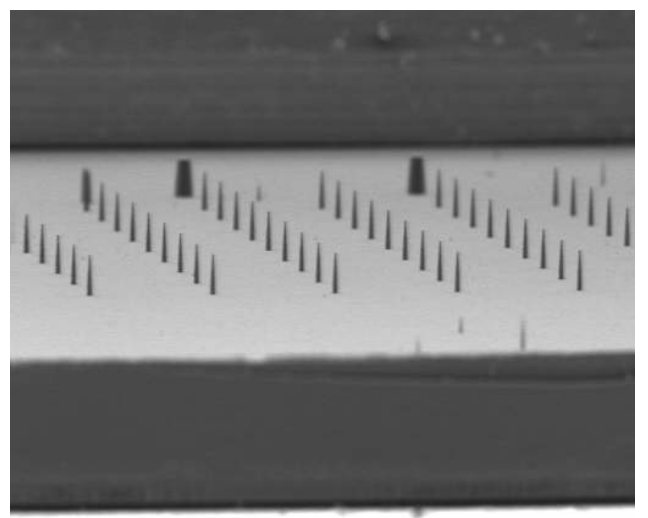

$\longdiv { 2 5 0 \mathrm { mm } }$

Figure 1. Si coupon with 81 microtips

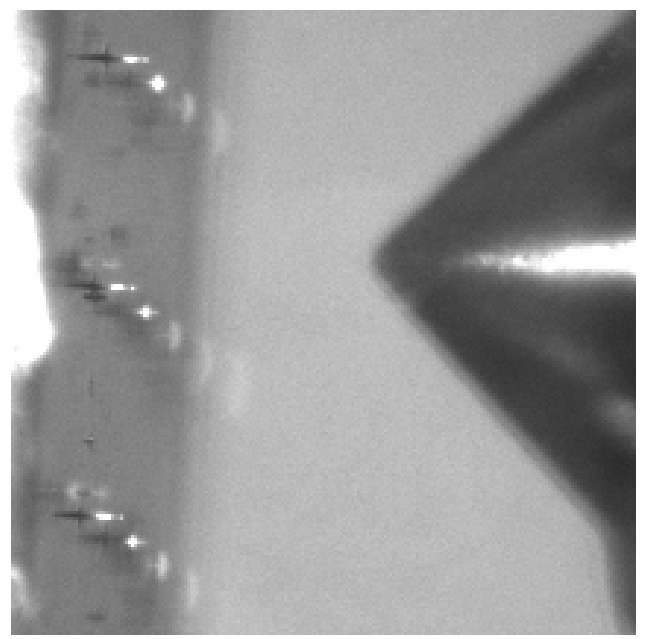

Fig. 2. Microtip coupon loaded in the analysis chamber. Each post is perfectly oriented toward the counter-electrode

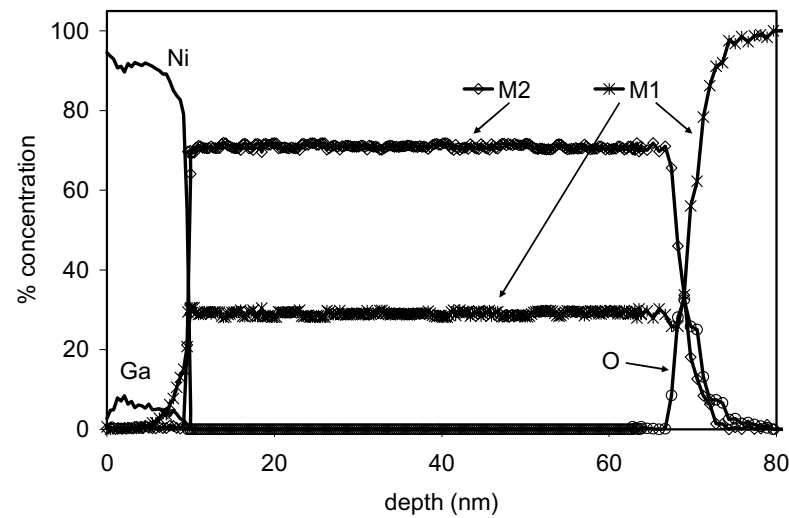

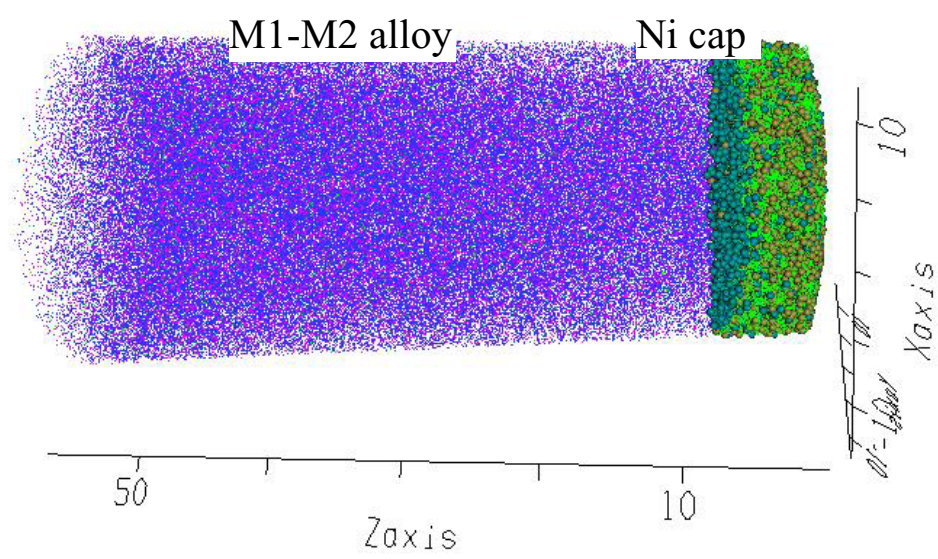

Fig. 3. 3-D image of the analyzed stack. The Ni-cap (green), Ga penetration (orange spheres), M1-oxide layer (light blue spheres) and M1-M2 alloy (blue and purple dots) are apparent.

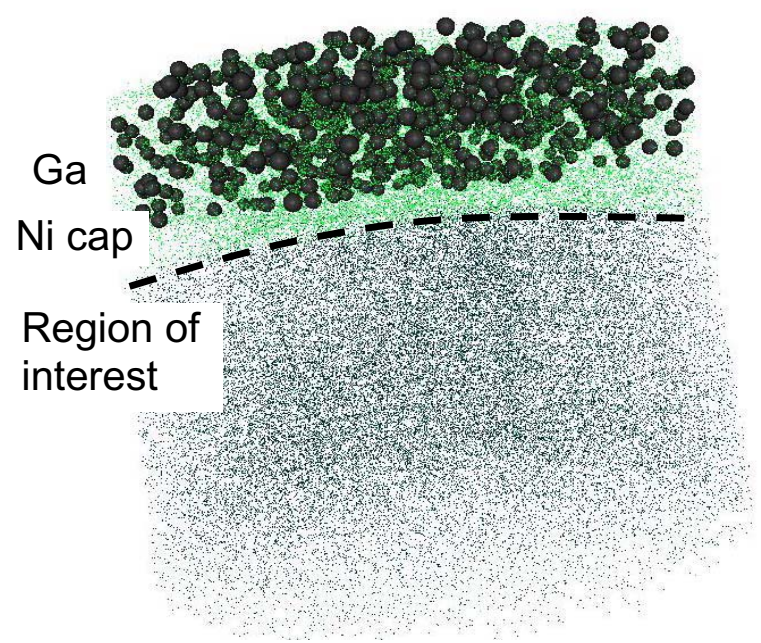

Fig. 4. Close-up 3-D image showing the Ga penetration into the Ni cap.

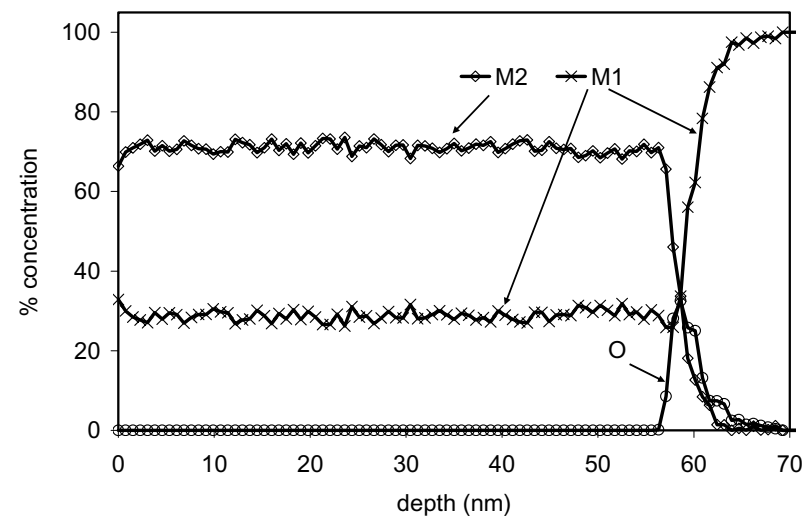

Figs. 5 1-D concentration profile of stack from pre-sharpened (left) and flat-top (right) microtip. The stack region of interests are statistically the same. 